\title{
Pedestrian/Bicyclist Limb Motion Analysis from 110-Car TASI Video Data for Autonomous Emergency Braking Testing Surrogate Development
}

\author{
Rini Sherony \\ TEMA \\ Renran Tian, Stanley Chien, Li Fu, and Yaobin Chen \\ Indiana University Purdue University \\ Hiroyuki Takahashi \\ Toyota Motor Corporation
}

\begin{abstract}
Many vehicles are currently equipped with active safety systems that can detect vulnerable road users like pedestrians and bicyclists, to mitigate associated conflicts with vehicles. With the advancements in technologies and algorithms, detailed motions of these targets, especially the limb motions, are being considered for improving the efficiency and reliability of object detection. Thus, it becomes important to understand these limb motions to support the design and evaluation of many vehicular safety systems. However in current literature, there is no agreement being reached on whether or not and how often these limbs move, especially at the most critical moments for potential crashes. In this study, a total of 832 pedestrian walking or cyclist biking cases were randomly selected from one large-scale naturalistic driving database containing 480,000 video segments with a total size of 94TB, and then the 832 video clips were analyzed focusing on their limb motions. We modeled the pedestrian/bicyclist limb motions in four layers: (1) the percentages of pedestrians and bicyclists who have limb motions when crossing the road; (2) the averaged action frequency and the corresponding distributions on when there are limb motions; (3) comparisons of the limb motion behavior between crossing and non-crossing cases; and (4) the effects of seasons on the limb motions when the pedestrians/bicyclists are crossing the road. The results of this study can provide empirical foundations supporting surrogate development, benefit analysis, and standardized testing of vehicular pedestrian/ bicyclist detection and crash mitigation systems.
\end{abstract}

CITATION: Sherony, R., Tian, R., Chien, S., Fu, L. et al., "Pedestrian/Bicyclist Limb Motion Analysis from 110-Car TASI Video Data for Autonomous Emergency Braking Testing Surrogate Development," SAE Int. J. Trans. Safety 4(1):2016, doi:10.4271/2016-01-1456.

\section{INTRODUCTION}

With the progress in Advanced Driver Assistance Systems (ADAS), more vehicles being manufactured today are equipped with pedestrian and bicyclist Autonomous Emergency Braking (AEB) systems because of their potential to reduce car-to-person crashes [1]. Compared to the car-to-car AEB systems, which have been well evaluated using standardized scenarios and processes $[\underline{2}, \underline{3}]$, the evaluation methods for car-to-pedestrian and car-to bicyclist AEB systems are still being formalized and are expected to be applied in the Euro NCAP in 2016 and 2018 [ $\underline{3}, \underline{4}]$. Similar standardized AEB tests may also be applied in the U.S. in the upcoming years. Under these circumstances, many efforts are made worldwide in finding the most critical car-to-person crash scenarios by developing the most representative pedestrian and bicyclist crash test surrogates $[\underline{3}, \underline{4}][\underline{11}$, $\underline{12}, \underline{13}, \underline{14}, \underline{15}, \underline{16}]$. Besides many key variables that researchers from different groups are trying to harmonize, there are some debates on the adoption of limb motions in the AEB crash test, especially when insufficient empirical data are available in the literature.
Limb motions for the pedestrian and bicyclists are important to increase the efficiency and accuracy of detection algorithms. For camera-based algorithms, various studies about general human detection $[\underline{5}]$, pedestrian detections $[\underline{6}, \underline{7}, \underline{8}]$, and human pose recognitions $[\underline{9}, \underline{10}, \underline{20}]$ all emphasize on the importance of human limb motions and corresponding posture changes, which provide strong clues in achieving better image-processing results. Some studies about pedestrian crash test surrogates designs [111, 12] also pointed out the changes of human body Radar Cross Section (RCS) with limb motions, and suggested introducing moving pedestrian crash dummies to simulate such RCS variations. The pedestrian limb motion refers to the movements of the arms and the legs, and the bicyclist limb motion refers to the movements of the legs as well as the pedals.

In the currently published studies about the pedestrian AEB crash test, some researchers use static pedestrian crash surrogate with fixed pre-defined postures [14], while some other researchers tried to develop moving crash dummies with natural full-body limb motions 
[16] and adopt different limb motions in the crash tests [15]. Similar differences are also taking place towards the bicyclist AEB crash test designs. With obvious advantages and disadvantages of these two methods in terms of cost and reliability, it is important to estimate the necessity of introducing limb motion in the AEB crash test based on the behaviors of pedestrians and bicyclists in the natural road environment. In other words, the best answers to include or not include limb motions in AEB evaluation process will be based on the existence and measures of these motions in the actual situations where they may have strong effects on AEB system performances.

As summarized in [17], most pedestrian behavior studies are mainly focused on step frequency, walking speeds, crossing decisionmaking, and gestures/postures. The total number of bicyclist limb motion related studies is limited. To our best knowledge, there is no study focusing on analyzing the pedestrian and bicyclist limb motion in order to support the AEB crash test design, especially towards the overall road environment. Thus in this study, we will try to analyze empirical limb motion data of pedestrians and bicyclists in the naturalistic road environment. The results of this study will provide details on pedestrians and bicyclists limb motions across all road locations and all types of man-vehicle encounters, including percentages of pedestrians/bicyclist with limb motions when crossing the road, the frequency of the limb motions, the differences between crossing and non-crossing cases, and any effects of different seasons on pedestrian limb motions.

The analysis is based on the TASI (Transportation Active Safety Institute) 110-car naturalistic driving video data set $[17, \underline{18}, \underline{19}]$. This dataset was constructed by the TASI researchers at Indiana University - Purdue University at Indianapolis. As one large scale driving data set, it contains the video files recording outward views of the road from 110-vehicles continuously for a period of one year. Multi-step data analysis was performed on the collected 94TB of data to detect all the encounters with pedestrians and bicyclists, assign scenario labels, and then calculate comprehensive road and driving features. For more details about the TASI 110-car naturalistic driving dataset, please refer to the earlier publications $[\underline{17}, \underline{18}, \underline{19}]$.

\section{METHODOLOGY FOR ANALYZING LIMB MOTIONS}

In this section, the methodology applied for limb motion analysis will be described. The research scope will be firstly discussed with research questions defined. The following video data analysis and limb motion data analysis are all based on the research question definitions. Then the process for limb motion video preparation as well as video analysis process is demonstrated. Finally, the limb motion data analysis method is presented.

\section{Description of the Research Questions}

As described earlier, this study is focusing on studying the limb motions of the pedestrians and bicyclists, specifically the arm motion of pedestrians and the leg motion of bicyclists, in order to support the AEB evaluations. The reasons to focus on the arm motion of the pedestrians include: (1) the pedestrians always have leg motions and the current debating point for AEB testing surrogate development is whether the arm motion is needed, and (2) when there are arm motions, the frequencies of the arm and leg motions are usually the same. For the bicyclists, considering that most of them do not have notable and regular arm motions, only the leg motions should be focused. Also because that it is still not clear whether or not the bicyclists usually have leg motions when crossing the streets, there are debates about if leg motions are necessary for AEB testing surrogate development. The limb motion data will be sampled from a large amount of pedestrian and bicyclist moving video database collected from the driver's view, and they are defined as:

Pedestrian limb motion refers to the arm swinging motion while walking. The whole arm (including the hand) needs to be clearly visible from the side of the pedestrian in order to be considered as swinging. The left arm and the right arm of the pedestrians are studied separately. One limb motion cycle (arm swing cycle) starts when the arm swings to the very backward position, and ends after the arm swing forward and back to the very backward position again.

Bicyclist limb motion refers to the leg kicking motions when moving the pedals. One limb motion cycle is the same as one pedal rotation cycle.

Since the scenarios for encounters between pedestrians and bicyclists are very complicated, it will be important to study the limb motions in the cases that are most relevant to the AEB system performance, and ignore the less relevant variables. In this study, we have defined five vehicle-pedestrian and vehicle-bicyclist encountering scenarios to study the corresponding limb motions, as illustrated in Figure 1, including:

1. Pedestrian crossing from both directions

2. Pedestrian walking with/against the traffic out of the roadway at both sides of the road

3. Bicyclist crossing from both directions

4. Bicyclist riding with/against the traffic in the roadway at both sides of the road

5. Bicyclist riding with/against the traffic out of the roadway at both sides of the road

In all of these selected scenarios, the driving direction of the vehicle is not limited because the limb motions of the pedestrians and bicyclists can be studied independently without concerning about the interactions with the vehicles. In the five selected scenarios, the two crossing scenarios are the most important ones mainly because that the limb motions will have most effect on image processing algorithms and radar RCS values when the pedestrians and bicyclists show their sides to the vehicle. Also, considering that the limb motions will be almost symmetric for the AEB system no matter which side of the pedestrian or bicyclist is seen, the left-to-right crossing and right-to-left crossing cases are combined together in these two crossing scenarios.

When the pedestrians/bicyclists are traveling along the road, they are mainly showing their front or back to the vehicle. In these situations the limb motions have less effect on object detection and AEB performances. Since the effects of limb motion in these cases on AEB 
evaluation are comparatively small no matter what the moving directions and locations are, along the road cases for pedestrians/ bicyclists are combined including both directions (with and against the traffic) and both locations (at the left and right side of the roads). However, since along the road cases are used as the control group to be compared with the crossing cases in terms of limb motions, we have separated the bicyclists riding along the road cases into two categories: in the roadway and out of the roadway, because these two situations happen quite frequently for the bicyclists with quite different behaviors associated with them.

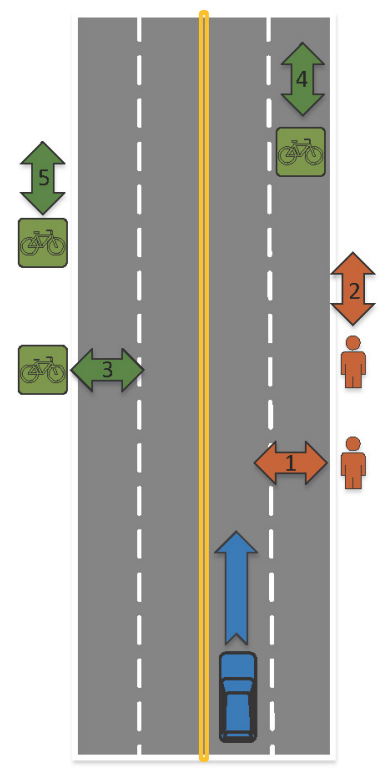

Figure 1. Four common vehicle-to-pedestrian and vehicle-to-bicyclist encounters when the vehicle is moving straight forward.

Please note that the pedestrian in-road walking with/against traffic scenario is not included in this study. This is mainly because of two reasons: (1) for pedestrian walking along the road situations, the effects of limb motions on AEB systems are not quite different no matter if the pedestrian is walking in-road or out-of-road; and (2) when walking along the road, the pedestrian behaviors are not as different as the bicyclists for in-road and out-of-road situations, and thus are not investigated in this study.

For the crossing cases, one important measure is the existence of the limb motion during the crossing period. In this study, the definition of crossing period can be described as:

The crossing period starts when the pedestrian enters the road, and ends when the pedestrian leaves the roads.

On the contrary, existence of limb motion or not for moving along the road cases is not a valid question, because there are no clear boundaries for these cases and the answer is totally relying on where the sample is selected and how long the duration is.

Due to the scope of this research, we only included one environment variable towards the limb motion analysis: seasons. At different seasons, the temperature varies significantly, and may thereby affect the limb motions. Such difference is expected to be more prominent for the pedestrians. This is because when the temperature changes, the pedestrians may wear different amount of clothing, hide their hands in the pockets, or take some other poses while walking. The seasons defined in this study rely on the monthly average temperatures in Indiana, where the naturalistic driving data collection was completed. The temperature data are collected from the website (www.weather.com) which defines the following three seasons:

Summer season includes May, June, July, August, and September with average high temperature above $70^{\circ} \mathrm{F}$.

Spring/Fall season includes March, April, October, and November with average high temperature from $50^{\circ} \mathrm{F}$ to $60^{\circ} \mathrm{F}$.

Winter season includes December, January, and February with average high temperature below $40^{\circ} \mathrm{F}$.

To summarize the discussions, we mainly have four detailed research questions as listed below, and the data analysis will be performed to try to answer all of these questions:

- What are the percentages of pedestrians/bicyclists who have limb motions while crossing the road?

- What are the average limb motion frequency and the corresponding distributions for the pedestrians and bicyclists crossing cases?

- What are the differences in limb motion behaviors between crossing and non-crossing cases for the pedestrians and the bicyclists?

- What are the effects of seasons on the limb motions when the pedestrians/bicyclists are crossing the road?

\section{Video Data Analysis Process}

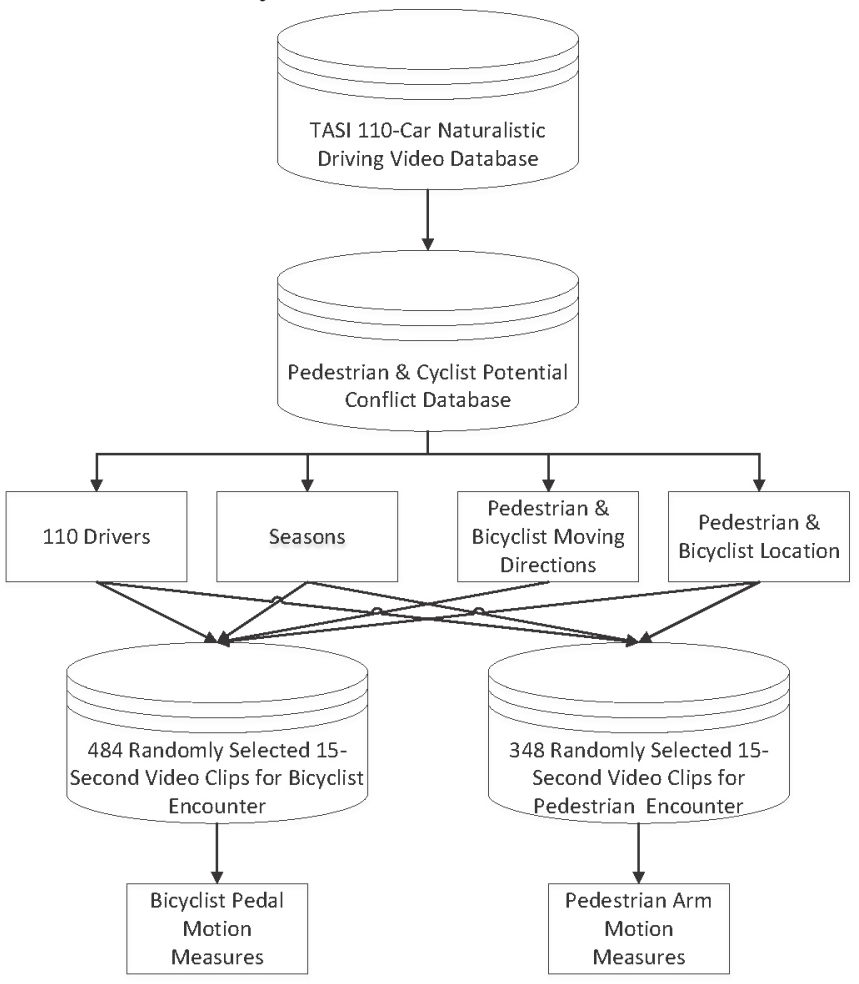

Figure 2. Pedestrian and bicyclist limb motion video data preparation process. 
In order to achieve the limb motion data for the pedestrians and bicyclists for the five selected scenarios, the limb motion video clips are first prepared following the process shown in figure 2. From the TASI 110-car naturalistic driving video database, the labeled pedestrian and bicyclist potential conflict database was first retrieved $[\underline{17}, \underline{18}, \underline{19}]$. This potential conflict database is composed of 15 -second video clips representing the cases marked as potential conflict with higher encountering risks [18], containing about 5000 pedestrian and bicyclist videos. Random samples are selected from this potential conflict database with four conditions:

1. The selected cases should cover more subjects from the 110 participated drivers;

2. The selected cases should be similarly distributed among all the months/seasons;

3. The selected cases should be similarly distributed among the crossing and moving along the road cases;

4. The selected cases should be similarly distributed for different in-road and out-of-road situations.

After applying the above-mentioned conditions, a total of 832 pedestrian and bicyclist video clips were randomly sampled from the TASI 110-car potential conflict database. The only criteria used for the sampling process is that pedestrian/bicyclist is not far away from the vehicle so that their limb motions are clearly seen from the video. Table 1 and table 2 shows the number of sampled pedestrian and bicyclist cases respectively under the selected five scenarios combining with the three defined seasons. The sample sizes are generally well balanced across all these categories for both pedestrian and bicyclist cases, and the only exception is the pedestrian walking along the road cases happening in the summer which only has 3 samples. However, since we only study the effects of seasons on pedestrian limb motions for crossing cases, the limited amount of samples in this category may not affect the analysis significantly.

Table 1. Number of pedestrian cases under different scenarios considering seasons and moving directions are presented.

\begin{tabular}{|l|l|l|l|}
\hline & Crossing & $\begin{array}{l}\text { Along the road } \\
\text { (Out of Road) }\end{array}$ & Total \\
\hline Spring/Fall & 80 & 79 & 159 \\
\hline Summer & 70 & 3 & 73 \\
\hline Winter & 71 & 45 & 116 \\
\hline Total & 221 & 127 & 348 \\
\hline
\end{tabular}

Table 2. Number of bicyclist cases under different scenarios considering seasons, moving locations, and moving directions are presented.

\begin{tabular}{|l|l|l|l|l|}
\hline & Crossing & $\begin{array}{l}\text { Along the road } \\
\text { (In Road) }\end{array}$ & $\begin{array}{l}\text { Along the road } \\
\text { (Out of Road) }\end{array}$ & Total \\
\hline Spring/Fall & 67 & 80 & 42 & 189 \\
\hline Summer & 73 & 72 & 81 & 226 \\
\hline Winter & 21 & 29 & 19 & 69 \\
\hline Total & 161 & 181 & 142 & 484 \\
\hline
\end{tabular}

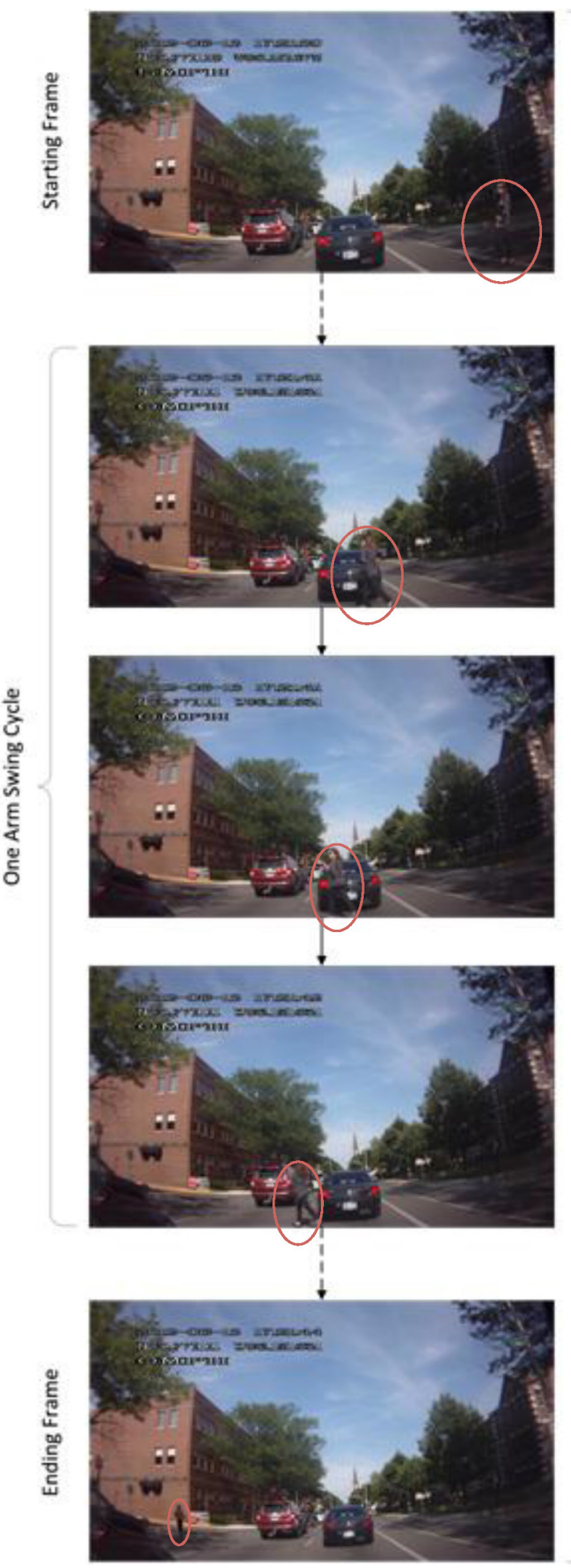

Figure 3. Video analysis example for achieving the limb motion measures towards pedestrian crossing cases. 
For each sampled pedestrian or bicyclist case, the video analysis was performed by trained data reductionists to achieve the limb motion data. One example of the video analysis process for a pedestrian crossing case is illustrated in Figure 3 . The first step of the video analysis is to determine the first frame and the last frame of the interested period. The interested period for one crossing case starts from the moment that the pedestrian enters the road (the first frame), and ends by the moment that the pedestrian leaves the road (the last frame). For the moving along the road cases, the interested period will be selected by the data reductionist as part of the video in which it is easiest to observe the limb motions of the pedestrians or bicyclists. Once the interested period with the starting/ending frames has been located in the video, the data reductionists will count the number of limb motion cycles during the period, as shown in Figure 3. For pedestrians, the arm swing cycles will be counted separately for left and right arms. For bicyclists, the bike pedal rotation cycles will be counted as an indication of the limb motions. Then, the limb motion frequency is calculated using the total number of motion cycles divided by the total duration to complete these cycles

\section{Limb Motion Data Analysis Method}

The collected limb motion data from the video analysis described above were analyzed towards the four research questions proposed. The data analysis mainly focused on the crossing cases to investigate the existence and distributions of limb motions. Then the movingalong-the-road cases were analyzed to compare with the crossing cases mainly in terms of the limb motion frequencies. Finally the effects of seasons on the limb motion measures for crossing cases were studied.

\section{RESULTS}

The analysis results are presented in this section corresponding to the four research questions.

\section{Existence of Limb Motions for Crossing Cases}

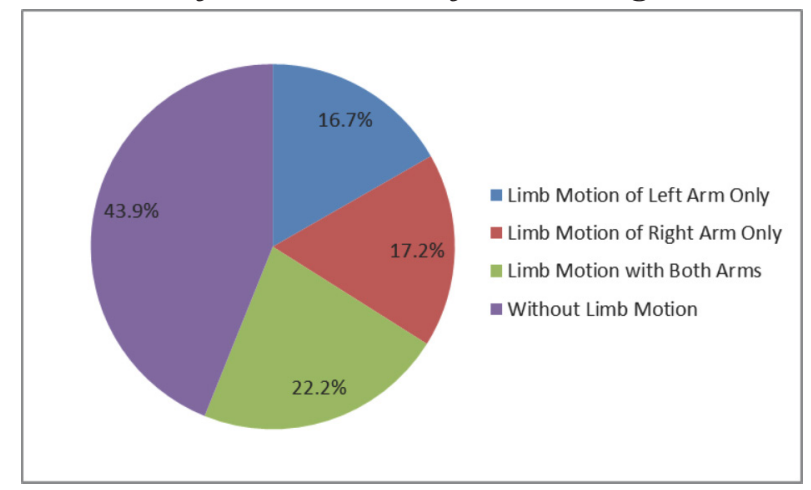

Figure 4. Percentages of pedestrian crossing cases with limb motions of left arm, right arm, and both arms as well as without limb motions $(n=221)$.

Figure 4 shows the percentages of pedestrian crossing cases with and without limb motions of the arms. In general, there are slightly less than the half of the pedestrians crossing the road without swinging their arms. For the pedestrians crossing with limb motions, about
$40 \%$ of them swing both arms. Then the rest of the pedestrians have almost equal chances to swing their left or right arms only while crossing the road.

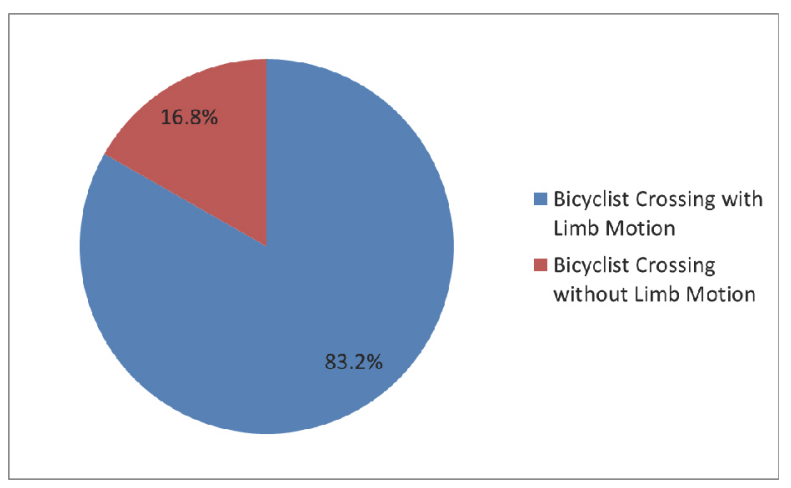

Figure 5. Percentages of bicyclists with and without limb motions when crossing the road $(\mathrm{n}=161)$.

For the bicyclist crossing cases, Figure 5 shows that majority of the bicyclists $(>80 \%)$ are pedaling during the road crossing period.

\section{Limb Motion Frequencies for Crossing Cases}

In this section, the frequencies of limb motions for pedestrian and bicyclist crossing cases are presented. The analysis is based on the crossing cases with limb motions. The total numbers of pedestrian and bicyclist crossing cases with limb motion are 122 and 134 respectively. The descriptive statistics for the pedestrian and bicyclist limb motion frequencies among all crossing cases are presented in Table 3. The mean value for pedestrian limb motion frequencies is 0.95 cycles/second, and the mean value for bicyclists is 0.85 cycles/ second.

Table 3. Descriptive statistics of the pedestrian and bicyclist limb motion frequencies among all crossing cases are presented.

\begin{tabular}{|l|l|l|l|l|l|l|}
\hline $\begin{array}{l}\text { Limb } \\
\text { Motion } \\
\text { Frequency } \\
(\mathrm{Hz})\end{array}$ & Mean & $\begin{array}{l}\text { Standard } \\
\text { Error }\end{array}$ & Median & $\begin{array}{l}\text { Standard } \\
\text { Deviation }\end{array}$ & Min & Max \\
\hline Pedestrian & 0.95 & 0.02 & 0.96 & 0.24 & 0.22 & 1.67 \\
\hline Bicyclist & 0.85 & 0.04 & 0.77 & 0.43 & 0.22 & 2.80 \\
\hline
\end{tabular}

$\underline{\text { Figures } 6}$ and $\underline{7}$ illustrate the distribution of pedestrian and bicyclist limb motion frequencies for all crossing cases respectively, as well as the cumulative percentages of all these cases with different frequencies. It can be inferred that the pedestrian limb motion frequency is mostly concentrating in the range from $0.9 \mathrm{HZ}$ to 1.2 $\mathrm{HZ}$, with a relatively symmetric distribution. For the bicyclist limb motion, the frequencies are mainly from $0.3 \mathrm{HZ}$ to $1.2 \mathrm{HZ}$, which account for $80 \%$ of all the cases. And the range from $0.7 \mathrm{HZ}$ to 0.9 $\mathrm{HZ}$ contains most of the cases. The distribution of the bicyclist limb motion frequencies skews to the left side remarkably. 


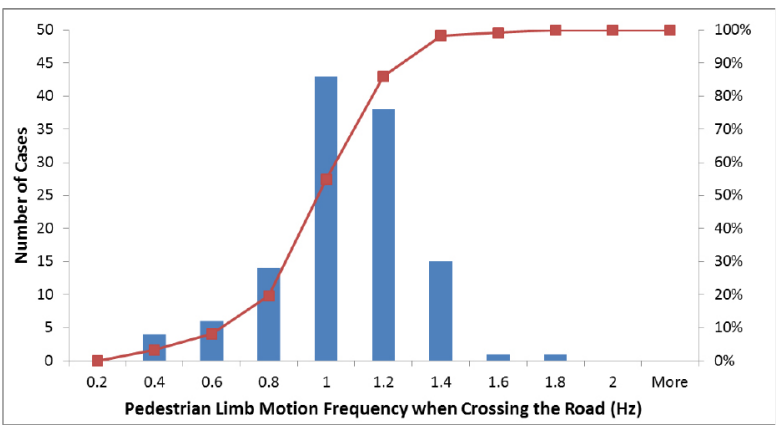

Figure 6. Distribution and cumulative percentages of the pedestrian limb motion frequencies when crossing the road $(\mathrm{n}=122)$.

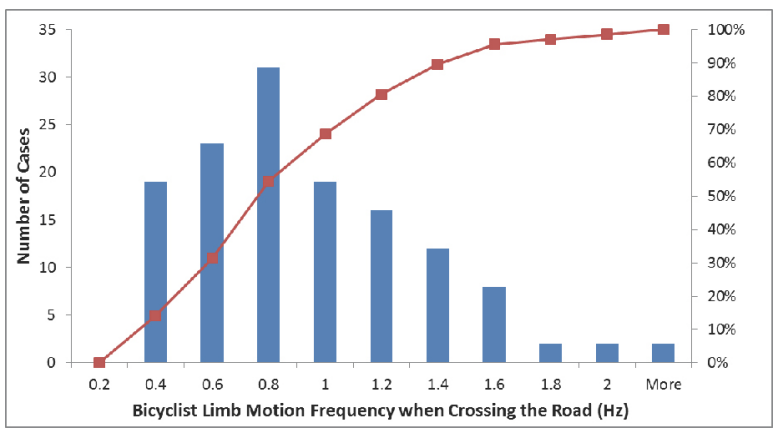

Figure 7. Distribution and cumulative percentages of the bicyclist limb motion frequencies when crossing the road $(\mathrm{n}=134)$.

\section{Comparison between Crossing and Non-Crossing Cases}

Figure 8 shows the comparison results for pedestrian crossing and non-crossing cases. During the data analysis, all the pedestrian cases with limb motions are combined together no matter if it is associated with single-arm or dual-arm limb motions. The results show that when there are limb motions, crossing pedestrians tend to swing their arms significantly faster $\left(t\right.$-test: $\left.t_{187}=3.36, p<0.001\right)$ comparing to those walking along/against the traffic out of the road. This result is consistent with the findings in [17], where the authors conclude that the pedestrian step frequencies for crossing cases are significantly faster than the step frequencies of walking outside of the road. In [17], the mean step frequency for crossing cases is $1.98 /$ second, and the mean step frequency for non-crossing cases is $1.71 /$ second. Considering that the pedestrian will make two steps within one arm swing cycle, the results are consistent between the two studies. This is understandable because the same database is used, although the two samples are independent from each other and was processed by different researchers.

Figure 9 shows the limb motion frequency comparison results for bicyclists at different scenarios. It is clear that the bicyclists pedal faster when riding with/against traffic in the road, and pedal slowly when crossing the road. Single-variable ANOVA test proves that the mean values of the three scenarios are significantly different $\left(F_{2,376}=6.95, p<0.01\right)$. More detailed $t$-test shows that the frequency for crossing cases is significantly smaller than moving along the road cases (in road) $\left(t_{276}=3.60, p<0.001\right)$, but is not significantly smaller than the moving along the road cases (out of road) $\left(t_{233}=1.09\right.$, $p=0.14>0.05$ ).

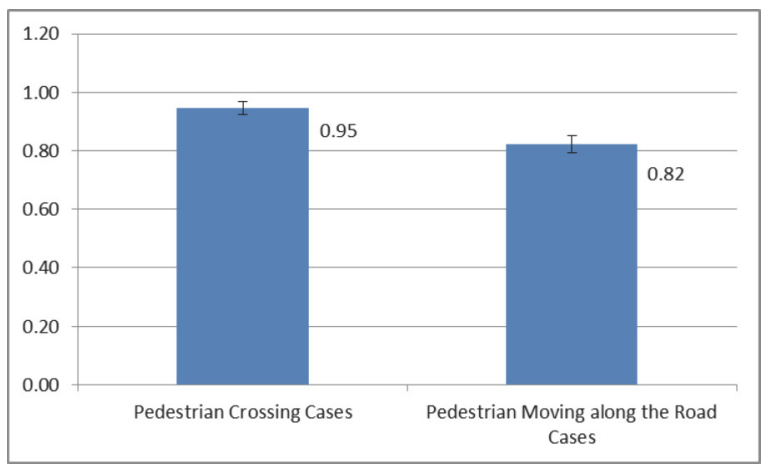

Figure 8. Comparison of the limb motion frequencies for pedestrian crossing and walking along the road cases with mean values and standard errors illustrated ( $\mathrm{n}=122$ and 67).

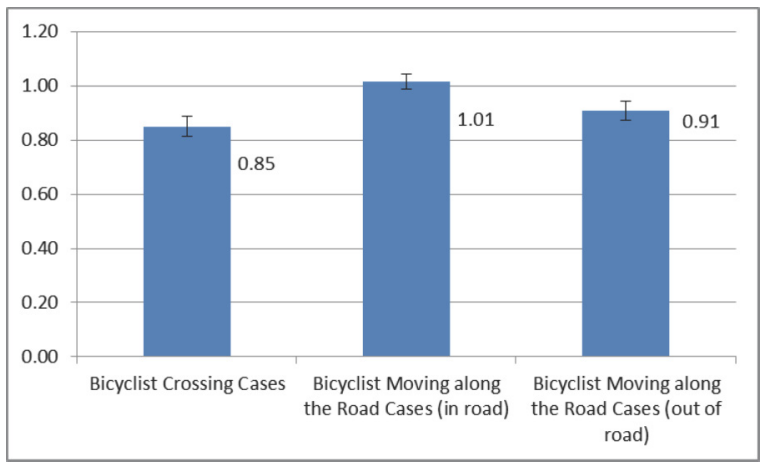

Figure 9. Comparison of the limb motion frequencies for bicyclist crossing, moving along the road (in road), and moving along the road (out of road) cases with mean values and standard errors illustrated $(\mathrm{n}=134,144$ and 101).

\section{Limb Motions for Crossing Cases at Different Seasons}

In this section, the effects of seasons on limb motions for crossing cases are studied. The main question is that at different seasons, whether or not there are same probabilities for the pedestrians and the bicyclists to have limb motions when crossing the road. Table 4 shows the crosstab table for all the pedestrian crossing cases among seasons and limb motion existences. The numbers in the table represent the total number of cases at different seasons that are with or without limb motions. From the table, we can tell that for spring/ fall and summer seasons, there are more pedestrians crossing the road with limb motions. The ratios of the number of cases with limb motions over the number of cases without limb motions are similar among these seasons. However, for the winter season, there is much less pedestrians crossing the road with limb motions. On the contrary, more of the pedestrians choose to cross the road without swinging their arms at all when the weather is cold. A chi-square analysis proves that the effects of seasons on pedestrian limb motion for crossing cases are statistically significant $(p<0.0001)$. 
Table 4. Crosstab table among seasons and limb motion existences for all the pedestrian crossing cases is presented.

\begin{tabular}{|c|c|c|c|c|}
\hline \multirow{2}{*}{\multicolumn{2}{|c|}{ Number of Cases }} & \multicolumn{2}{|c|}{ Limb Motion Existence } & \multirow{3}{*}{\begin{tabular}{|l} 
Total \\
80
\end{tabular}} \\
\hline & & No & Yes & \\
\hline \multirow{3}{*}{ Season } & Spring/Fall & 28 & 52 & \\
\hline & Summer & 20 & 50 & 70 \\
\hline & Winter & 49 & 22 & 71 \\
\hline \multicolumn{2}{|l|}{ Total } & 97 & 124 & 221 \\
\hline
\end{tabular}

Table 5 shows the crosstab table for all the bicyclist crossing cases among seasons and limb motion existences. The numbers in the table represent the total number of cases at different seasons that are with or without limb motions. From the table, we can tell that for all the seasons, there are more bicyclists crossing the road with limb motions. The ratios of the number of cases with limb motions over the number of cases without limb motions are similar among all the seasons. A chi-square analysis proves that the effects of seasons on bicyclist limb motion existences for crossing cases are not statistically significant ( $p=0.415>0.05)$.

Table 5. Crosstab table among seasons and limb motion existences for all the bicyclist-crossing cases is presented.

\begin{tabular}{|l|l|l|l|l|}
\hline \multirow{2}{*}{ Number of Cases } & \multicolumn{2}{l}{ Limb Motion Existence } & \multirow{2}{*}{ Total } \\
\cline { 3 - 6 } & No & Yes & 67 \\
\hline \multirow{3}{*}{ Season } & Spring/Fall & 14 & 53 & 73 \\
\cline { 2 - 5 } & Summer & 11 & 62 & 21 \\
\cline { 2 - 5 } & Winter & 2 & 19 & 161 \\
\hline Total & 27 & 134 & & \\
\hline
\end{tabular}

\section{SUMMARY/CONCLUSIONS}

In this study, we present some empirical results about pedestrian and bicyclist limb motions to support the surrogate development, benefit analysis, and standardized testing of vehicular pedestrian/bicyclist detection and crash mitigation systems. Relying on the large-scale TASI 110-car naturalistic driving video database, we have sampled 832 pedestrian and bicyclist video clips for limb motion analysis, covering the scenario variables of moving directions, moving locations, and seasons. These sampled videos were analyzed manually to count the numbers of limb motion cycles. Then data analysis was performed to answer four research questions. The percentages of crossing cases with and without limb motions for pedestrians and bicyclists are reported respectively, with distributions of the limb motion frequencies presented. It is evident that the pedestrian moved arms in $56 \%$ of the cases and bicyclists moved legs in $83 \%$ of the cases when crossing and seen by the vehicles. At the same time, we have concluded that the pedestrians tend to have higher limb motion frequencies when crossing the road, while the bicyclists tend to have lower limb motion frequencies while crossing, when compared to moving along-the-road cases. It has also been found in this study that although the seasons do not significantly affect the limb motions of bicyclists, the cold temperature during winter season significantly reduce the chances for the pedestrians to have limb motions while crossing. When over $2 / 3$ of all pedestrians cross the road swinging their arms during warmer weather, only $1 / 3$ of them will do that during the cold winter.

\section{REFERENCES}

1. Rosén, E., Källhammera, J., Erikssona, D., Nentwicha, M., Fredrikssona, R., and Smith, K., "Pedestrian injury mitigation by autonomous braking", Accident Analysis and Prevention, 42(6):1949-1957,2010, doi: 0.1016/j.aap.2010.05.018

2. Hulshof W., Knight, I., Edwards, A., Avery, M., and Grover, C., "Autonomous Emergency Braking Test Results", In Proceedings of the 23rd International Technical Conference on the Enhanced Safety of Vehicles (ESV), Seoul, Republic of Korea, May 27 - 30, 2013.

3. Schram, R., Williams, A., and Van Ratingen, M., "Implementation of Autonomous Emergency Braking (AEB), the Next Step in Euro NCAP's Safety Assessment", In Proceedings of the 23rd International Technical Conference on the Enhanced Safety of Vehicles (ESV), Seoul, Republic of Korea, May 27 - 30, 2013.

4. Rigling, A., "Testing and Assessment of AEB Systems for Consumer Protection", In 5th International Munich Chassis Symposium, pp 623632, July 192014

5. Dalal, N., Triggs, B., and Schmid, C., "Human Detection Using Oriented Histograms of Flow and Appearance", In European Conference on Computer Vision (ECCV '06), Lecture Notes in Computer Science (LNCS), May 2006, Graz, Austria, vol. 3952, pp: 428-441.

6. Kilicarslan, M., and Zheng, J. Y., "Detecting Walking Pedestrians from Leg Motion in Driving Video", In IEEE 17th International Conference on Intelligent Transportation Systems (ITSC), October 8-11, 2014. Qingdao, China, pp: 2924 - 2929, doi: 10.1109/ITSC.2014.6958159

7. Curio, C., Edelbrunner, J., Kalinke, T., Tzomakas, C., and von Seelen, W., "Walking Pedestrian Recognition", IEEE Transaction on Intelligent Transportation System, 1(3):155 - 163, September 2000, doi: $\underline{10.1109 / 6979.892152}$

8. Shashua, A., Gdalyahu, Y., and Hayun, G., "Pedestrian Detection for Driving Assistance Systems: Single-Frame Classification and System Level Performance", In IEEE Intelligent Vehicles Symposium, June 14 17, 2004, pp: 1-6, doi: 10.1109/IVS.2004.1336346

9. Shotton, J., Fitzgibbon, A., Cook, M., Sharp, T., Finocchio, M., Moore, R., Kipman, A., and Blake, A., "Real-time Human Pose Recognition in Parts from Single Depth Images", Communications of the ACM, 56(1):116-124, 2013, DOI: $10.1145 / 2398356.2398381$

10. Ouyang, W., Chu, X., and Wang, X., "Multi-source Deep Learning for Human Pose Estimation", In IEEE Conference on Computer Vision and Pattern Recognition (CVPR), Columbus, OH, USA, June 23 - 28, 2014, pp: $2337-2344$

11. Lemmen, P., Stoll, J., Bergelt, U., Seiniger, P., Wisch, M., Bartels, O., Schubert, E., Kunert, M., Knight, I., Brookes, D., Ranovona, M., Okawa, T., Domsch, C., and Schaller, T., "Evaluation of Pedestrian Targets for Use In Autonomous Emergency Brake System Testing - A Report from the Harmonisation Platform 2 Dealing with Test Equipment," In Proceedings of the 23rd International Technical Conference on the Enhanced Safety of Vehicles (ESV), Seoul, Republic of Korea, May 27 30, 2013.

12. Schubert, E., Kunert, M., Menzel, W., Fortuny-Guasch, J., Chareau, J.-M., "Human RCS Measurements and Dummy Requirements for the Assessment of Radar based Active Pedestrian Safety Systems", In 14th International Radar Symposium (IRS), Dresden, Germany, June 19-21, 2013, pp: 752 - 757.

13. Lenard, J., Russell, D., Avery, M., Weekes, A., Zuby, D., and Kuehn, M., "Typical Pedestrian Accident Scenarios for the Testing of Autonomous Emergency Braking Systems," in the 22nd International Technical Conference on the Enhanced Safety of Vehicles (ESV), Washington, D.C., June 13-16, 2011.

14. Seiniger, P., Hellmann A., Bartels O., Wisch M., and Gail J., "Test Procedures and Results for Pedestrian AEB Systems", In the 24th International Technical Conference on the Enhanced Safety of Vehicles (ESV) Gothenburg, Sweden, June 8-11, 2015.

15. Chien, S., Yi, Q., Good, D., Gholamjafari, A., Chen, Y., and Sherony, R., "Method of Selecting Test Scenarios for Pedestrian Forward Looking Pre-Collision System Evaluation," SAE Technical Paper 2014-01-0163, 2014, doi: $10.4271 / 2014-01-0163$. 
16. Yi, Q., Chien, S., Brink, J., Chen, Y., Li, L., Good, D., Chen, C. C., and Sherony, R., "Mannequin Development for Pedestrian Pre-Collision System evaluation", In 17th IEEE International Conference on Intelligent Transportation Systems (ITSC), October 8-11 2014, pp: 1626 - 1631, Qingdao, China, doi: 10.1109/ITSC.2014.6957926

17. Tian, R., Du, E. Y., Yang, K., Jiang, P., Jiang, F., Chen, Y., Sherony, R., and Takahashi, H., "Pilot Study on Pedestrian Step Frequency in Naturalistic Driving Environment", In IEEE Intelligent Vehicles Symposium (IV), June 23-26 2013, pp: 1215 - 1220, Gold Coast, QLD, Australia, doi: 10.1109/IVS.2013.6629632

18. Tian, R., Li, L., Yang, K., Chien, S., Chen, Y., and Sherony, R., "Estimation of the Vehicle-Pedestrian Encounter/Conflict Risk on the Road based on TASI 110-Car Naturalistic Driving Data Collection", In IEEE Intelligent Vehicles Symposium, June 8-11 2014, pp: 623 - 629, Dearborn, MI, USA, doi: 10.1109/IVS.2014.6856599

19. Tian, R., Li, L., Yang, K., Jiang, F., Chen, Y., and Sherony, R., "SingleVariable Scenario Analysis of Vehicle-Pedestrian Potential Crash Based on Video Analysis Results of Large-Scale Naturalistic Driving Data", Digital Human Modeling. Applications in Health, Safety, Ergonomics and Risk Management: Ergonomics and Health, Volume 9185 of the series Lecture Notes in Computer Science, pp: 295-304, 2015, doi: $\underline{10.1007 / 978-3-319-21070-4 \_30}$

20. Yuasa, H., Nakanishi, M., Mochida, T., Yamada, N., and Nakai, M., "Research into Evaluation Method for Pedestrian Precollision System", In Proceedings of the 23rd International Technical Conference on the Enhanced Safety of Vehicles (ESV), Seoul, Republic of Korea, May 27 30, 2013.

\section{CONTACT INFORMATION}

\section{Dr. Renran Tian}

Transportation Active Safety Institute Indiana University Purdue University Indianapolis

723 West Michigan Street, SL 160, Indianapolis, IN 46202-5160

Phone: (317) 278-8717

rtian@iupui.edu

Rini Sherony

Collaborative Safety Research Center

Toyota Motor Engineering \& Manufacturing North America

Phone: (734) 995-7116

rini.sherony@tema.toyota.com

\section{ACKNOWLEDGMENTS}

The authors would like to acknowledge Toyota's Collaborative Safety Research Center for their support of this research.

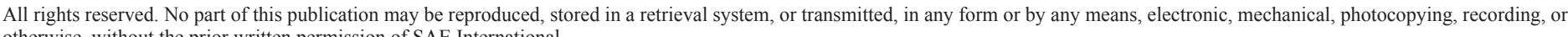
otherwise, without the prior written permission of SAE International. 